\title{
Direct imaging of curve-crossing dynamics in IBr by attosecond transient absorption spectroscopy
}

\author{
Yuki Kobayashi, ${ }^{1,} \quad$ Kristina F. Chang, ${ }^{1}$ Tao Zeng, ${ }^{2}$ \\ Daniel M. Neumark, ${ }^{1,3, \dagger}$ and Stephen R. Leone ${ }^{1,3,4,+}$ \\ ${ }^{1}$ Department of Chemistry, University of California, Berkeley, California 94720, USA \\ ${ }^{2}$ Department of Chemistry, Carleton University, Ottawa, Ontario K1S5B6, Canada \\ ${ }^{3}$ Chemical Sciences Division, Lawrence Berkeley National Laboratory, Berkeley, California 94720, USA \\ ${ }^{4}$ Department of Physics, University of California, Berkeley, California 94720, USA
}

(Dated: February 21, 2019)

The electronic character of photoexcited molecules can abruptly change at avoided crossings and conical intersections. Here, we report direct spectroscopic imaging of such nonadiabatic processes in a prototype molecule, iodine monobromide ( $\mathrm{IBr}$ ), using extreme-ultraviolet (XUV) attosecond transient absorption spectroscopy. A few-femtosecond visible pulse resonantly excites the $\mathrm{B}\left({ }^{3} \Pi_{0^{+}}\right)$state and the accompanying dissociation dynamics are tracked by an attosecond XUV pulse that simultaneously probes the I- $4 d$ and $\mathrm{Br}-3 d$ core-level absorption edges. Direct comparison with quantum mechanical simulations unambiguously identifies the core-level absorption features associated with adiabatic and diabatic channels at the avoided crossing that is accessed by one-photon and two-photon visible-excitation processes. The results delineate rapid switching of valence-orbital vacancies from $\sigma$ to $\pi / \pi$ ensuing at the avoided crossing. (118/125 words) 
The simple picture of a photoexcited molecule evolving on a single reactive potential surface while smoothly changing its physical character is invalid when nonadiabatic interactions induce coupling between neighboring electronic states [1-3]. Avoided crossings and conical intersections arise, and the implications of these nonadiabatic regimes have been of fundamental interest in molecular physics since the seminal Landau-Zener model pioneered in the 1930s $[4,5]$. It is now widely accepted that nonadiabatic interactions play key roles in broad classes of photochemical reactions, such as photoisomerization in the retinal chromophore [6] and photostability of DNA bases against ultraviolet radiation [7]. Laser-based control of nonadiabatic processes represents a pivotal milestone in fully understanding complex photochemical reactions $[8,9]$.

Despite these successes, the real-time observation of the coupled dynamics between electrons and nuclei in nonadiabatic regimes remains elusive [10]. Conceptually, rapid switching of electronic character takes place between nonadiabatically-coupled states in synchrony with nuclear motion. Experimentally, few-femtosecond time resolution is required to probe such short-lived dynamics, and the degeneracy of the coupled states makes it challenging to obtain state-resolved information. Several experimental methods have been applied to this problem [11-15], including novel highharmonic spectroscopy [16] and ultrafast electron diffraction [17]. Recent theoretical studies predicted that transient absorption spectroscopy in the x-ray/extreme-ultraviolet (XUV) range offers a unique and powerful route to image nonadiabatic dynamics, with core-to-valence transitions capturing the marked reorganization of valence-electron densities in nonadiabatic regimes [18, 19]. When combined with high-harmonic-generation-based attosecond light sources, the method has the potential to ultimately attain subfemtosecond probing time resolution [20].

Here, we report on attosecond XUV transient absorption probing of valence-electron dynamics accompanying visible-light excitation of iodine monobromide (IBr), a prototype molecule for avoided crossings $[21,22]$. Our primary focus is the direct spectroscopic imaging of electroniccharacter switching that ensues at the avoided crossing. An outline of the experiment is depicted in Fig. 1A. Ultrafast visible-light excitation is performed in the pump step $(\lambda=530 \mathrm{~nm}, \Delta \lambda=70$ $\mathrm{nm}, 8 \mathrm{fs}, 15 \mu \mathrm{J}$, Fig. 1B), which selectively excites the neutral electronic states of IBr and minimizes the unfavorable ionization effects that arise in more typical experiments where intense near-infrared excitation is used [23]. In the probe step after a delay time $\tau$, an attosecond XUV pulse produced by high-harmonic generation is introduced (45-72 eV, 200 as, Fig. 1C), which encodes the time evolution of the photoexcited processes in the characteristic core-to-valence absorption signals. A novel feature of this experiment is full tracking of the photofragment electronic states by means of the XUV probe pulse that accesses the two core-level absorption edges: the $\mathrm{N}_{4,5}$ edge ( $4 d$ orbitals) 
of iodine and the $\mathrm{M}_{4,5}$ edge ( $3 d$ orbitals) of bromine. In the time-resolved measurements, the differential optical density $(\Delta \mathrm{OD})$ is recorded as a function of delay time; $\triangle \mathrm{OD}$ is defined as a logarithmic ratio of the transmitted XUV spectra with and without the visible-pump pulse.

Figure 1D shows the potential energy curves of IBr. Systematic investigation into the electronic structures of diatomic interhalogens including $\mathrm{IBr}$ was conducted by Child [21, 22]. Multiple avoided crossings are present in diatomic interhalogens because of the strong spin-orbit couplings of the halogen atoms and the absence of $g-u$ symmetry for heteronuclear molecules. Two physical pictures are invoked to describe the dynamics in nonadiabatic regimes [24]: in a diabatic picture, electronic states conserve their character as they move along the reaction coordinate, whereas in an adiabatic picture, electronic states are eigenstates of the electronic Hamiltonian and are subject to switching of electronic character at the avoided crossing. The visible-pump pulse excites an attractive $\mathrm{B}\left({ }^{3} \Pi_{0^{+}}\right)$state, which diabatically correlates with the spin-orbit excited $\left.\mathrm{I}^{2}{ }^{2} P_{3 / 2}\right)+$ $\mathrm{Br} *\left({ }^{2} P_{1 / 2}\right)$ asymptote. The $\mathrm{B}\left({ }^{3} \Pi_{0^{+}}\right)$state undergoes an avoided crossing $\left(R_{c}=3.3 \AA\right)$ with a repulsive $\mathrm{Y}\left(0^{+}\right)$state. The nonadiabatic coupling at the $\mathrm{B} / \mathrm{Y}$ avoided crossing enables the system to adiabatically transfer from the attractive $\mathrm{B}\left({ }^{3} \Pi_{0^{+}}\right)$potential to the repulsive $\mathrm{Y}\left(0^{+}\right)$potential and dissociate into the ground $\mathrm{I}\left({ }^{2} P_{3 / 2}\right)+\operatorname{Br}\left({ }^{2} P_{3 / 2}\right)$ asymptote (Fig 1D, inset). The nonadiabatic coupling is of intermediate strength for $\mathrm{IBr}$, and the probabilities for the photoexcited molecule diabatically remaining on the attractive $\mathrm{B}\left({ }^{3} \Pi_{0^{+}}\right)$potential (diabatic channel) or adiabatically transferring to the repulsive $\mathrm{Y}\left(0^{+}\right)$potential (adiabatic channel) is approximately 3:1 [25]. In the experiments, resonance-enhanced two-photon processes are also observed for the visible-pump pulse, which reaches a peak-field intensity of $5 \times 10^{13} \mathrm{~W} / \mathrm{cm}^{2}$. An electronic-structure analysis reveals a large transition dipole moment ( $>3$ Debye) associated with the $\mathrm{B} \rightarrow \mathrm{Y}$ transition. The $\mathrm{Y}\left(0^{+}\right)$state undergoes an inner avoided crossing $\left(R_{c}=2.8 \AA\right)$ with the higher $\mathrm{Z}\left(0^{+}\right)$state, which diabatically correlates with the $\mathrm{I}^{*}\left({ }^{2} P_{1 / 2}\right)+\operatorname{Br}\left({ }^{2} P_{3 / 2}\right)$ asymptote. The $\mathrm{B} \rightarrow \mathrm{Z}$ transition is also possible but of less importance ( $20 \%$ compared with $\mathrm{B} \rightarrow \mathrm{Y}$ ), and is not considered further here. See supplementary materials for more details [23].

We first analyze the transient-absorption spectrum of the atomic dissociation products recorded at a 215-245 fs delay time (Fig. 2A). Iodine and bromine fragment atoms each exhibit three absorption lines in the I $4 d \rightarrow 5 p$ and $\mathrm{Br} 3 d \rightarrow 4 p$ series: ${ }^{2} P_{3 / 2} \rightarrow{ }^{2} D_{5 / 2}(45.9,64.5 \mathrm{eV}),{ }^{2} P_{1 / 2} \rightarrow$ ${ }^{2} D_{3 / 2}(46.7,65.1 \mathrm{eV})$, and ${ }^{2} P_{3 / 2} \rightarrow{ }^{2} D_{3 / 2}(47.6,65.6 \mathrm{eV})[26,27]$. A group of weak absorption signals at 55-58 eV correspond to the I $4 d \rightarrow n p(n>5)$ Rydberg series [28]. The production ratio between the $\mathrm{Br}$ and $\mathrm{Br}^{*}$ atoms calculated from the XUV absorption amplitudes is $\mathrm{Br} / \mathrm{Br}=3.3$, which is in reasonable agreement with a previous measurement at $525-\mathrm{nm}$ excitation $(\mathrm{Br} / \mathrm{Br}=3.0$ 
[25]). At the same time, a sizable signal from the I atom is observed, yielding a production ratio of $\mathrm{I} / \mathrm{I}=0.5$. The appearance energy of the $\mathrm{I}$ atom $(2.8 \mathrm{eV})$ is higher than the center photon energy of the visible-pump pulse $(2.3 \mathrm{eV})$, thus indicating that both one-photon and two-photon processes are excited in the experiments.

Fig. 2B shows transient absorption spectra as a function of delay time. The measurements are performed from -16 fs to 245 fs delay time with a step size of $1.5 \mathrm{fs}$. The pump-on/pumpoff XUV spectra are each averaged over 200 laser pulses to calculate $\Delta O D$. The excited-state absorption $(\Delta \mathrm{OD}>0))$ is shown in bright colors, and the ground-state bleach $(\Delta \mathrm{OD}<0))$ is shown in gray shades. The ground-state bleach features, both in the I- $4 d$ and $\mathrm{Br}-3 d$ windows, exhibit signatures of vibrational coherences in the ground $X\left({ }^{1} \Sigma_{0^{+}}\right)$state (isotopic averages: $\omega_{e}=$ $268 \mathrm{~cm}^{-1}, \omega_{e} x_{e}=0.8 \mathrm{~cm}^{-1}, T=125$ fs [29]). In previous studies, vibrational coherences in the neutral ground states were launched by strong-field ionization [30, 31]. Here, the excitation process is a resonant single-photon transition, and unique nodal structures are newly observed in the oscillating absorption bands. We performed quantum wave-packet calculations and simulated the core-level absorption spectra, and the observed features are reproduced by taking coherences in the $v=0,1$, and 2 vibrational states (Fig. S3). The mechanism for the overtone $(v=2)$ excitation in the neutral ground state is attributed to a stimulated Raman process enhanced by resonant visible-light coupling between the $\mathrm{X}\left({ }^{1} \Sigma_{0^{+}}\right)$and $\mathrm{B}\left({ }^{3} \Pi_{0^{+}}\right)$states. See supplementary materials for more details [23].

Figures $3 \mathrm{~A}$ and $\mathrm{B}$ show the details of the excited-state absorption in the $\mathrm{I}-4 d$ and $\mathrm{Br}-3 d$ windows, respectively. The early-time signals (0-50 fs) exhibit sweeping shifts to lower photon energy, which map the dissociative motion of the photoexcited molecule on the $\mathrm{B}\left({ }^{3} \Pi_{0^{+}}\right)$potential. The evolution of the electronic states at the $\mathrm{B} / \mathrm{Y}$ avoided crossing is imprinted in the subsequent temporal window (50-80 fs, dashed boxes); the corresponding absorption traces, taken at 6 fs intervals $(50,56,62$, 68, 74, and $80 \mathrm{fs})$, are shown in Figs. $3 \mathrm{C}$ and $\mathrm{D}$. The $\mathrm{I}^{*}(46.7 \mathrm{eV})$ and $\mathrm{Br}^{*}(65.1 \mathrm{eV})$ signals are almost invariant with respect to delay time; they conceivably originate from two-photon processes occurring on a shorter time scale, and this assignment is verified by ab-initio simulations as will be shown later. The other signals (denoted by arrows with labels diabatic/adiabatic) exhibit dramatic variations both in the absorption amplitude and energy. The diabatic channel leads to the I $+\mathrm{Br}^{*}$ asymptote (Fig. 1D) and the associated wave packet conserves its electronic character during passage through the avoided crossing. In the Br-3d window (Fig. 3D), the signal exhibiting continuous shifts to lower photon energy is indeed converging to the $\mathrm{Br}^{*}$ absorption line, and it is assigned to the diabatic channel. In the adiabatic channel, on the other hand, the 
photoexcited molecule changes electronic character and proceeds to the ground $\mathrm{I}+\mathrm{Br}$ asymptote (Fig. 1D). In the experimental results, the $\mathrm{Br}$ absorption line suddenly emerges at a separate photon energy accompanied by no energy shift (Fig 3D). This discontinuous development of the $\mathrm{Br}$ signal is a manifestation of the new electronic character acquired from the neighboring $\mathrm{Y}\left(0^{+}\right)$ state. In the I-4d window (Fig. 3C) the XUV absorption signals associated with the diabatic and adiabatic channels are not as widely separated since the same I fragment is produced in both channels. Nonetheless, the continuous shifts to lower photon energy and the sudden emergence of the converged I absorption line are distinctly resolved, leading to consistent assignments of the diabatic and adiabatic channels.

To gain more detailed insight into the transitory molecular features, we perform ab-initio simulations of the core-to-valence absorption spectra [23]. The valence and core-excited electronic structures are computed by spin-orbit generalized multi-configurational quasi-degenerate perturbation theory (SO-GMC-QDPT) [32, 33], and from these the XUV absorption strengths are obtained. Nonadiabatic dissociation dynamics are computed fully quantum mechanically by numerically solving the time-dependent Schrödinger equation. The initial wave packets are taken to be in the Franck-Condon region of the $\mathrm{B}\left({ }^{3} \Pi_{0^{+}}\right)$state (one-photon process) or on the $\mathrm{Y}\left(0^{+}\right)$state (two-photon process) to model the relevant visible-light excitations.

Simulated results for the one-photon process are shown in Figs. 3E and F. The sweeping energy shifts in the early-time window are successfully reproduced in the simulation. The $\mathrm{B}\left({ }^{3} \Pi_{0^{+}}\right)$ state in the Franck-Condon region has a $\left[\sigma^{2} \pi^{4} \pi{ }^{3} \sigma^{1}\right]$ configuration and is probed by the corelevel transitions to the $\pi$ and $\sigma$ orbitals. The observed absorption lines reflect the multiplet structures of the core-excited states that originate from the spin-orbit couplings in the $\mathrm{I}-4 d, \mathrm{Br}-3 d$, and $\pi$ orbitals (Fig. S6). Some features are broadened and less pronounced in the experiments compared to the simulations, which is attributed to the spectral width of the visible-pump pulse and the nuclear wave-packet motion on the repulsive core-excited potentials. The simulated traces in the temporal window for the $\mathrm{B} / \mathrm{Y}$ avoided crossing (50-80 fs) are shown in Figs. $3 \mathrm{G}$ and $\mathrm{H}$, with assignments of the diabatic and adiabatic channels given. The continuous (diabatic) and discontinuous (adiabatic) evolution of the XUV absorption signals is reproduced, providing clear confirmation of the experimental signal assignments.

The origin of the $\mathrm{I}^{*}$ and part of the $\mathrm{Br}^{*}$ signals is verified by the two-photon simulations shown in Figs. 3I and J. The simulated I* absorption signal (Fig. 3I) matches the experimental result (Fig. 3A) both in time and absorption energy. Passage through the $\mathrm{Y} / \mathrm{Z}$ avoided crossing (Fig. 1D) is calculated to occur in $25 \mathrm{fs}$; these early-time dynamics are not as clearly resolved in 
the experiments due to the spectral broadening from the fast dissociative motion on the repulsive $\mathrm{Y}$ and $\mathrm{Z}$ potentials. In Fig. $\mathrm{S} 7$ calculated absorption strengths of the $\mathrm{Y}\left(0^{+}\right)$and $\mathrm{Z}\left(0^{+}\right)$states are provided for future reference. The $\mathrm{Br}^{*}$ atom is produced in the two-photon process by way of the wave packet adiabatically transferring from the repulsive $\mathrm{Y}\left(0^{+}\right)$potential to the attractive $\mathrm{B}\left({ }^{3} \Pi_{0^{+}}\right)$potential at the $\mathrm{B} / \mathrm{Y}$ avoided crossing (Fig. 1D). As such, in the simulation, the switching of electronic character causes the abrupt emergence of part of the $\mathrm{Br}^{*}$ signal (Fig. $3 \mathrm{~J}$ ), and this is resolved in the experiments (Fig. 3B). Importantly, the dissociations in the two-photon processes are complete on a shorter time scale ( $60 \mathrm{fs}$ total) than compared to the one-photon process, and their contributions in the time window for the $\mathrm{B} / \mathrm{Y}$ avoided crossing are mostly independent of time.

The electronic-structure information imprinted in the XUV absorption spectra at the $\mathrm{B} / \mathrm{Y}$ avoided crossing is further examined based on the orbital energies and electron configurations. Figure 4 shows the energies of the active molecular orbitals as a function of internuclear distance computed at the level of multi-configurational self-consistent field (MCSCF). The energy differences between the core and valence orbitals are closely tied to the observed absorption energies. The exact description of the electronic states requires multiconfigurational treatments and inclusion of spinorbit couplings, as is done in the SO-GMC-QDPT calculations [23]. The energies of the I-4d and Br-3d orbitals are almost invariant throughout the reaction coordinate. This insensitivity to internuclear distance indicates that the observed energy shifts in the diabatic channel (or no shift in the adiabatic channel) reflect the energy variations in the valence orbitals. As for the trends among the valence orbitals, the energy gradients quantified at the $\mathrm{B} / \mathrm{Y}$ avoided crossing $\left(R_{c}=3.3\right.$ $\AA$, shaded area) are +1.5 and $-1.7 \mathrm{eV} / \AA$ for $\sigma$ and $\sigma$, respectively, but only +0.3 and $-0.2 \mathrm{eV} / \AA$ for $\pi$ and $\pi$, respectively. These internuclear-distance dependences indicate that the $\pi$ and $\pi$ orbitals are effectively no longer contributing to the variation of the potential energies when the wave packet reaches the avoided crossing. A more direct clue to the electronic-character switching is obtained from the analysis of the electronic configurations [34-36]. Just before the avoided crossing, the $\mathrm{B}\left({ }^{3} \Pi_{0^{+}}\right)$state is a mixed configuration of $\left[\sigma^{2} \pi^{4} \pi^{3} \sigma^{1}\right]$ and $\left[\sigma^{2} \pi^{3} \pi^{4} \sigma{ }^{1}\right]$; a single vacancy lies in $\sigma$, and the other vacancy is distributed between $\pi$ and $\pi$. After the photoexcited molecule adiabatically transfers to the repulsive $\mathrm{Y}\left(0^{+}\right)$potential, the main configuration changes to $\left[\sigma^{2} \pi^{3} \pi{ }^{3} \sigma^{2}\right]$, where the $\sigma$ orbital is fully occupied and the two vacancies lie in the $\pi$ and $\pi$ orbitals. The results point to a conclusion that at the $\mathrm{B} / \mathrm{Y}$ avoided crossing, the valence unoccupied-orbital character switches from $\sigma$ to $\pi / \pi$, giving rise to the emergence of new corelevel absorption signals with no further energy shifts, reflecting the energy invariance of the $\pi$ and 
$\pi$ orbitals versus internuclear distance.

The role of the $\mathrm{B} / \mathrm{Y}$ avoided crossing in the visible-band dynamics of $\mathrm{IBr}$ is usually measured via the final product ratio of $\mathrm{Br}$ and $\mathrm{Br} *$. Here, the attosecond XUV probe enables direct imaging of valence-electron dynamics during the passage through the avoided crossing, with core-level absorption sensitively encoding the energies and occupations of the valence orbitals. The valenceelectronic structure not only determines the strength of chemical bonds, but also governs the reactivity of a molecule as represented by the frontier-orbital theory; unraveling the nonadiabatic evolution of valence-electronic structures in dissociation or collision processes will extend the fundamental knowledge of physical chemistry. Application of ultrafast $\mathrm{x}$-ray/XUV light sources in such explorations of chemical dynamics is an actively advancing field of research $[37,38]$, and the present results serve as a valuable benchmark for future investigations of more complex molecules. 


\section{REFERENCES}

ykoba@berkeley.edu

’dneumark@berkeley.edu

*srl@berkeley.edu

[1] G. A. Worth and L. S. Cederbaum, Annual Review of Physical Chemistry 55, 127 (2004).

[2] B. G. Levine and T. J. Mart'inez, Annual Review of Physical Chemistry 58, 613 (2007).

[3] M. P. Bircher, E. Liberatore, N. J. Browning, S. Brickel, C. Hofmann, A. Patoz, O. T. Unke, T. Zimmermann, M. Chergui, P. Hamm, U. Keller, M. Meuwly, H.-J. Woerner, J. Vaníček, and U. Rothlisberger, Structural Dynamics 4, 061510 (2017).

[4] L. D. Landau, Phys. Z. Sowjetunion 2, 46 (1932).

[5] C. Zener and R. H. Fowler, R. Soc. London Ser. A 137, 696 (1932).

[6] D. Polli, P. Altoè, O. Weingart, K. M. Spillane, C. Manzoni, D. Brida, G. Tomasello, G. Orlandi, P. Kukura, R. A. Mathies, M. Garavelli, and G. Cerullo, Nature 467, 440 (2010).

[7] T. Schultz, E. Samoylova, W. Radloff, I. V. Hertel, A. L. Sobolewski, and W. Domcke, Science 306, 1765 (2004).

[8] B. J. Sussman, D. Townsend, M. Y. Ivanov, and A. Stolow, Science 314, 278 (2006).

[9] M. E. Corrales, J. González-Vázquez, G. Balerdi, I. R. Solá, R. De Nalda, and L. Bañares, Nature Chemistry 6, 785 (2014).

[10] S. R. Leone, C. W. McCurdy, J. Burgdörfer, L. S. Cederbaum, Z. Chang, N. Dudovich, J. Feist, C. H. Greene, M. Ivanov, R. Kienberger, U. Keller, M. F. Kling, Z.-H. Loh, T. Pfeifer, A. N. Pfeiffer, R. Santra, K. Schafer, A. Stolow, U. Thumm, and M. J. J. Vrakking, Nature Photonics 8, 162 (2014).

[11] S. Takeuchi, S. Ruhman, T. Tsuneda, M. Chiba, T. Taketsugu, and T. Tahara, Science 322, 1073 (2008).

[12] M. C. E. Galbraith, S. Scheit, N. V. Golubev, G. Reitsma, N. Zhavoronkov, V. Despré, F. Lépine, A. I. Kuleff, M. J. J. Vrakking, O. Kornilov, H. Köppel, and J. Mikosch, Nature Communications 8, 1018 (2017).

[13] A. von Conta, A. Tehlar, A. Schletter, Y. Arasaki, K. Takatsuka, and H. J. Wörner, Nature Communications 9, 3162 (2018).

[14] A. E. Boguslavskiy, O. Schalk, N. Gador, W. J. Glover, T. Mori, T. Schultz, M. S. Schuurman, T. J. Mart'inez, and A. Stolow, The Journal of Chemical Physics 148, 164302 (2018).

[15] H. Timmers, X. Zhu, Z. Li, Y. Kobayashi, M. Sabbar, M. Hollestein, M. Reduzzi, T. J. Mart'inez, D. M. Neumark, and S. R. Leone, (Submitted).

[16] H. J. Wörner, J. B. Bertrand, B. Fabre, J. Higuet, H. Ruf, A. Dubrouil, S. Patchkovskii, M. Spanner, Y. Mairesse, V. Blanchet, E. Mével, E. Constant, P. B. Corkum, and D. M. Villeneuve, Science 334, 
208 (2011).

[17] J. Yang, X. Zhu, T. J. A. Wolf, Z. Li, J. P. F. Nunes, R. Coffee, J. P. Cryan, M. Gühr, K. Hegazy, T. F. Heinz, K. Jobe, R. Li, X. Shen, T. Veccione, S. Weathersby, K. J. Wilkin, C. Yoneda, Q. Zheng, T. J. Martinez, M. Centurion, and X. Wang, Science 361, 64 (2018).

[18] S. P. Neville, V. Averbukh, S. Patchkovskii, M. Ruberti, R. Yun, M. Chergui, A. Stolow, and M. S. Schuurman, Faraday Discuss. 194, 117 (2016).

[19] S. P. Neville, M. Chergui, A. Stolow, and M. S. Schuurman, Phys. Rev. Lett. 120, 243001 (2018).

[20] F. Krausz and M. Ivanov, Rev. Mod. Phys. 81, 163 (2009).

[21] M. S. Child and R. B. Bernstein, The Journal of Chemical Physics 59, 5916 (1973).

[22] M. S. Child, Molecular Physics 32, 1495 (1976).

[23] See supplementary materials.

[24] T. Van Voorhis, T. Kowalczyk, B. Kaduk, L.-P. Wang, C.-L. Cheng, and Q. Wu, Annual Review of Physical Chemistry 61, 149 (2010).

[25] E. Wrede, S. Laubach, S. Schulenburg, A. Brown, E. R. Wouters, A. J. Orr-Ewing, and M. N. R. Ashfold, The Journal of Chemical Physics 114, 2629 (2001).

[26] L. Nahon, P. Morin, and F. C. Farnoux, Physica Scripta 1992, 104 (1992).

[27] G. O’Sullivan, C. McGuinness, J. T. Costello, E. T. Kennedy, and B. Weinmann, Phys. Rev. A 53, 3211 (1996).

[28] L. Nahon and P. Morin, Phys. Rev. A 45, 2887 (1992).

[29] R. E. Willis and W. W. Clark, The Journal of Chemical Physics 72, 4946 (1980).

[30] E. R. Hosler and S. R. Leone, Phys. Rev. A 88, 023420 (2013).

[31] Z. Wei, J. Li, L. Wang, S. T. See, M. H. Jhon, Y. Zhang, F. Shi, M. Yang, and Z.-H. Loh, Nature Communications 8, 735 (2017).

[32] T. Zeng, The Journal of Chemical Physics 146, 144103 (2017).

[33] Y. Kobayashi, T. Zeng, D. M. Neumark, and S. R. Leone, Structural Dynamics 6, 014101 (2019).

[34] S. Patchkovskii, Phys. Chem. Chem. Phys. 8, 926 (2006).

[35] R. Li, C. Wei, Q. Sun, E. Sun, M. Jin, H. Xu, and B. Yan, Journal of Quantitative Spectroscopy and Radiative Transfer 133, 271 (2014).

[36] T. Matsuoka and S. Yabushita, The Journal of Physical Chemistry A 119, 9609 (2015).

[37] M. Chergui and A. H. Zewail, ChemPhysChem 1o, 28 (2009).

[38] P. M. Kraus, M. Zürch, S. K. Cushing, D. M. Neumark, and S. R. Leone, Nature Reviews Chemistry 2, 82 (2018).

[39] D. J. Seery and D. Britton, The Journal of Physical Chemistry 68, 2263 (1964).

\section{ACKNOWLEDGEMENTS}

Funding: This work, Y.K., and K.F.C were supported by the National Science Foundation 
(NSF) (No. CHE-1660417). Y.K., K.F.C., D.M.N., and S.R.L. also acknowledge the funding from the US Army Research Office (ARO) (No. W911NF-14-1-0383). Development of calculation codes by T.Z. was supported by the Natural Sciences and Engineering Research Council (NSERC) of Canada for research funding (No. RGPIN-2016-06276) and Carleton University for start-up Grant (No. 186853). Some of the computations by Y.K. were performed using workstations at the Molecular Graphics and Computation Facility (MGCF) at UC Berkeley, which is supported by the National Institutes of Health (NIH) (Grant No. S10OD023532). Y.K. also acknowledges financial support from the Funai Overseas Scholarship.

Author contributions: Y.K., D.M.N., and S.R.L. conceived the experiments. Y.K. and K.F.C. performed the measurements. T.Z. designed the computational codes. Y.K. performed the calculations. Y.K. analyzed the results and wrote the manuscript with inputs from all authors.

Competing interests: The authors declare no competing interests.

Data and materials availability: All data is available in the manuscript or the supplementary materials. 


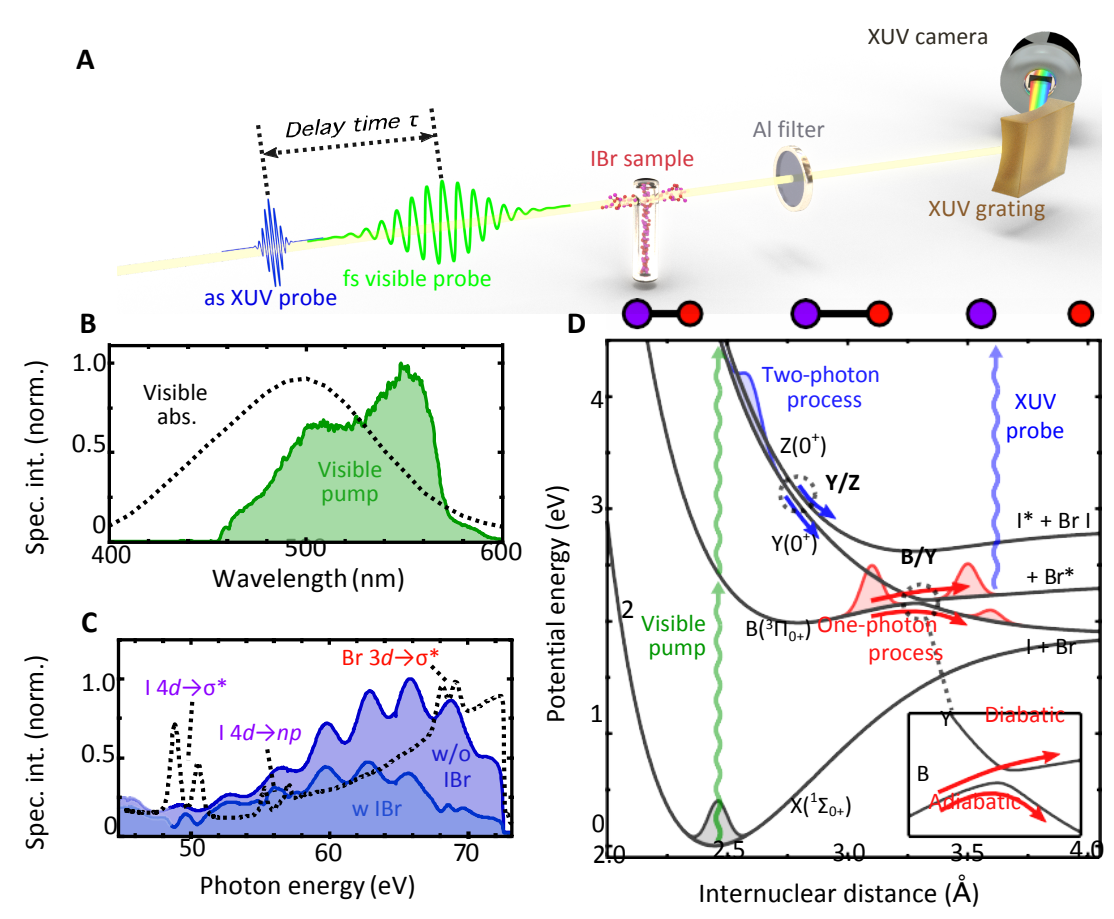

FIG. 1. Outline of experiment. (A) Illustration of the experimental setup. (B) Spectrum of the visiblepump pulse (green area), and visible-absorption band of IBr (black dashed curve) taken from ref. [39]. (C) Spectra of the XUV-probe pulse recorded with (light blue area) and without (dark blue area) the IBr sample, and the optical density (black dashed curve) calculated from the two spectra. The I- $4 d$ and Br-3 $d$ core-level edges are probed by the XUV pulse. (D) Adiabatic potential energy curves of IBr, with the visible and XUV excitation pathways marked by vertical arrows. The red and blue wave packets represent the dissociation pathways from one-photon and two-photon processes, respectively. Avoided crossings are formed between the $\mathrm{B}$ and $\mathrm{Y}$ states $\left(R_{c}=3.3 \AA\right)$ and $\mathrm{Z}$ and $\mathrm{Y}$ states $\left(R_{c}=2.8 \AA\right)$. The inset shows the adiabatic and diabatic channels at the $\mathrm{B} / \mathrm{Y}$ avoided crossing. 


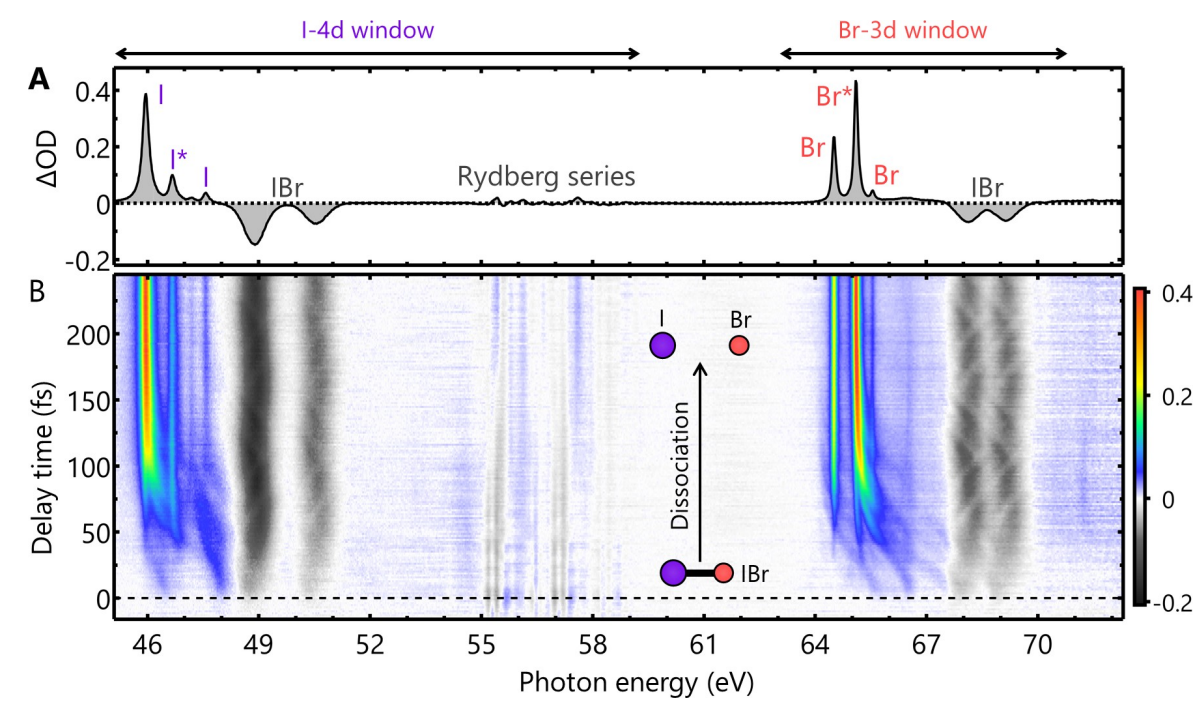

FIG. 2. Double-element XUV transient absorption spectra. (A) Transient absorption spectrum of the dissociation products recorded in the 215-245 fs delay range. (B) Delay-time resolved transient absorption spectra. At delay time zero, the visible-pump pulse initiates the dissociation processes, and excitedstate signal (rainbow colors) as well as ground-state bleach (gray and black colors) emerge. The dissociation proceeds toward the positive delays. 

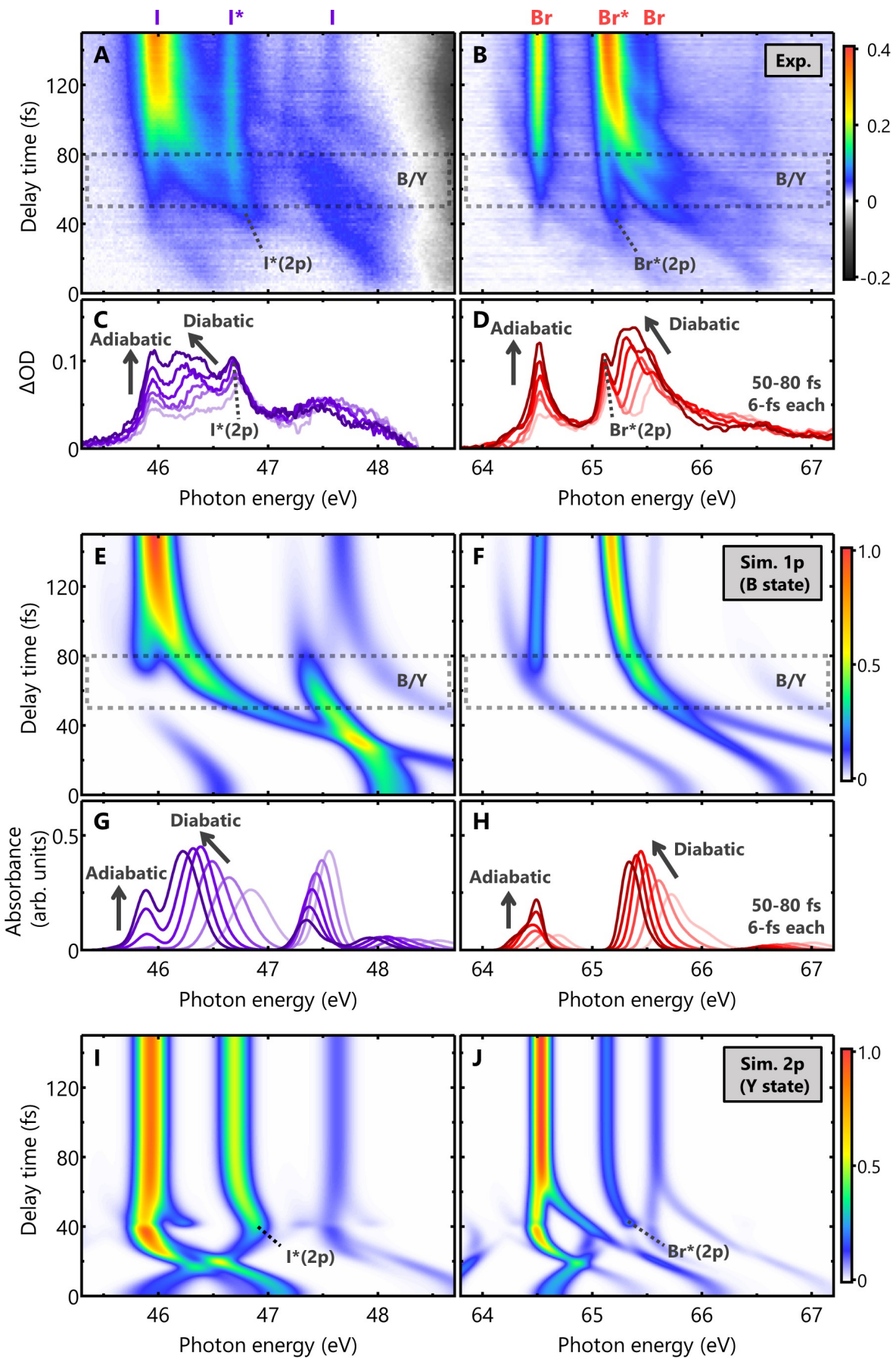

FIG. 3. Experimental and simulated XUV absorption spectra of avoided-crossing dynamics. (A and B) Experimental absorption spectra in the (A) I- $4 d$ and (B) Br-3d windows, respectively The temporal window for the $\mathrm{B} / \mathrm{Y}$ avoided crossing is highlighted by dashed boxes. The I* and $\mathrm{Br}$ * signals originating from the two-photon (2p) process is also marked. (C and D) Absorption spectra taken at delay times of $50,56,62,68,74$, and 80 fs from the experimental results. The assignments at the adiabatic and diabatic channels at the $\mathrm{B} / \mathrm{Y}$ avoided crossing are given by arrows. ( $\mathbf{E}$ to $\mathbf{H}$ ) One-photon simulation results shown for the comparison with the experimental results (A) to (D). (I and $\mathbf{J})$ Two-photon simulation results that reproduce the fast emergences of the $\mathrm{I}^{*}$ and $\mathrm{Br}^{*}$ signals. 


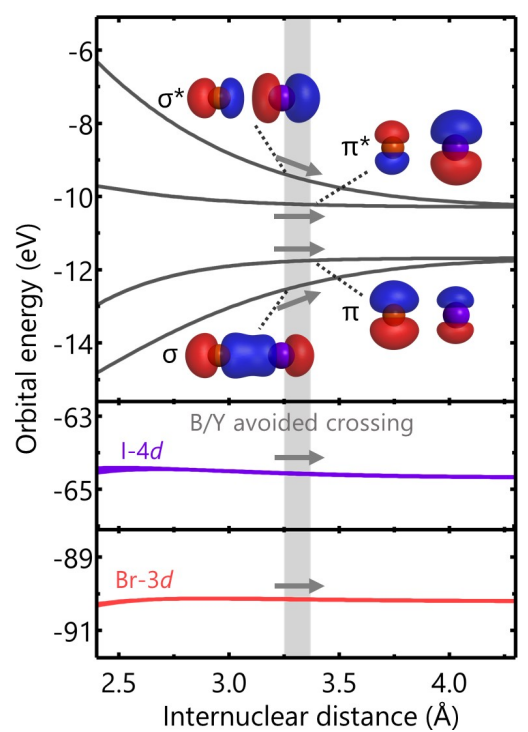

FIG. 4. Orbital energies of IBr as a function of internuclear distance. The energy difference between the core and valence orbitals are tied to the observed absorption energies. The $\sigma$ and $\sigma$ orbitals have long-range attractive and repulsive interactions, whereas the energies of the $\pi$ and $\pi$ orbitals are almost invariant around the $\mathrm{B} / \mathrm{Y}$ avoided crossing (gray area). The $3 \mathrm{D}$ plots are the valence molecular orbitals obtained at $R=3.3 \AA$. 